\title{
QUEEN'S
UNIVERSITY
BELFAST
}

\section{TOF diagnosis of laser accelerated, high-energy protons}

Scuderi, V., Milluzzo, G., Doria, D., Alejo, A., Amico, A. G., Booth, N., Cuttone, G., Green, J. S., Kar, S., Korn, G., Larosa, G., Leanza, R., Martin, P., McKenna, P., Padda, H., Petringa, G., Pipek, J., Romagnani, L., Romano, F., ... Borghesi, M. (2020). TOF diagnosis of laser accelerated, high-energy protons. Nuclear Instruments and Methods in Physics Research, Section A: Accelerators, Spectrometers, Detectors and Associated Equipment, 978, [164364]. https://doi.org/10.1016/j.nima.2020.164364

Published in:

Nuclear Instruments and Methods in Physics Research, Section A: Accelerators, Spectrometers, Detectors and Associated Equipment

\section{Document Version:}

Peer reviewed version

\section{Queen's University Belfast - Research Portal:}

Link to publication record in Queen's University Belfast Research Portal

\section{Publisher rights}

Copyright 2020 Elsevier

This manuscript is distributed under a Creative Commons Attribution-NonCommercial-NoDerivs License

(https://creativecommons.org/licenses/by-nc-nd/4.0/), which permits distribution and reproduction for non-commercial purposes, provided the author and source are cited.

\section{General rights}

Copyright for the publications made accessible via the Queen's University Belfast Research Portal is retained by the author(s) and / or other copyright owners and it is a condition of accessing these publications that users recognise and abide by the legal requirements associated with these rights.

Take down policy

The Research Portal is Queen's institutional repository that provides access to Queen's research output. Every effort has been made to ensure that content in the Research Portal does not infringe any person's rights, or applicable UK laws. If you discover content in the Research Portal that you believe breaches copyright or violates any law, please contact openaccess@qub.ac.uk. 


\title{
TOF diagnosis of laser accelerated, high-energy protons
}

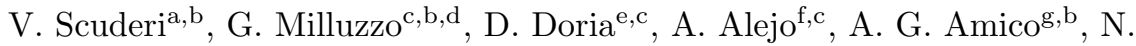 \\ Booth $^{\mathrm{h}}$, G. Cuttone ${ }^{\mathrm{b}}$, J.S. Green ${ }^{\mathrm{h}}$, S. Kar ${ }^{\mathrm{c}}$, G. Korn ${ }^{\mathrm{a}}$, G. Larosa ${ }^{\mathrm{b}}$, R. \\ Leanza $^{\mathrm{b}, \mathrm{d}}$, P. Martin ${ }^{\mathrm{c}}$, P. McKenna ${ }^{\mathrm{i}}$, H. Padda ${ }^{\mathrm{i}}$, G. Petringab,d ${ }^{\mathrm{b}}$, J. Pipek ${ }^{\mathrm{b}}$, L.

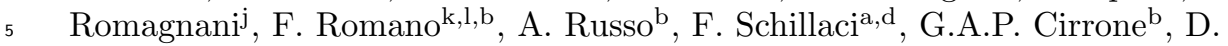 \\ Margarone $^{\mathrm{c}, \mathrm{a}}$, M. Borghesi ${ }^{\mathrm{c}}$ \\ ${ }^{a}$ Institute of Physics ASCR, v.v.i. (FZU), ELI-Beamlines Project, Prague, Czech Republic \\ ${ }^{b}$ INFN-Laboratori Nazionali del Sud (LNS-INFN), Via S Sofia 62, Catania, Italy \\ ${ }^{c}$ School of Mathematics and Physics, Queens University Belfast, Belfast, U.K. \\ ${ }^{d}$ Physics and Astronomy Department, University of Catania, Catania, Italy \\ ${ }^{e}$ National Institute for Physics and Nuclear Engineering, ELI-NP, Str. Reactorului, nr. 30, \\ P.O.Box MG-6, Bucharest-Magurele, Romania \\ ${ }^{f}$ University of Oxford, Department of Physics, Clarendon Laboratory, Parks Road Oxford \\ OX1 3PU \\ 15 MedAustron Ion Therapy Center, Wiener Neustadt, Austria \\ ${ }^{h}$ Central Laser Facility, STFC Rutherford Appleton Laboratory, Didcot OX11 OQX, U.K. \\ ${ }^{i}$ Department of Physics, SUPA, University of Strathclyde, Glasgow G4 ONG, U.K. \\ ${ }^{j}$ LULI, Ecole Polytechnique, CNRS, CEA, UPMC, 91128 Palaiseau, France \\ ${ }^{k}$ Istituto Nazionale di Fisica Nucleare, Sezione di Catania, 95125 Catania, Italy \\ ${ }^{l}$ National Physical Laboratory, Hampton Rd, Teddington, Middlesex, UK
}

\begin{abstract}
Significant challenges in the detection of laser-accelerated ions result from the high flux $\left(10^{10}-10^{12}\right.$ ions/pulse) and the short bunch duration which are intrinsic to laser-driven sources. The development of diagnostic techniques able to operate in real-time and on a high-rep basis is a key step towards multidisciplinary applications of such non-conventional beams. Real time diagnosis of the main beam parameters for high-energy protons accelerated by the Vulcan Petawatt (VULCAN-PW) laser system has been performed using an on line diagnostics based on the Time of Flight (TOF) technique and the use of diamond detectors. Proton energy spectra have been measured for energies exceeding $30 \mathrm{MeV}$. The results show that the TOF method employing state-of-the-art detectors is a robust real-time diagnostics, able to operate efficiently under the harsh conditions occuring with kJ-class, PW laser systems, and offering the possibility to monitor on a shot-by-shot basis the main beam parameters of high intensity proton bunches for energies up to the $100 \mathrm{MeV}$ level.
\end{abstract}

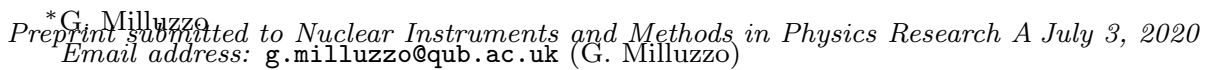


Keywords: Laser-driven ions, ELIMED beam line, Geant4, Multidisciplinary applications

\section{Introduction}

The recent development of high repetition rate $(1-10 \mathrm{~Hz})$ laser systems with power up to the PW regime enables investigation of new mechanisms of laserplasma ion acceleration [1]. The established acceleration mechanism known as TNSA (Target Normal Sheath Acceleration) [2-4] as well as emerging schemes, including RPA (Radiation Pressure Acceleration) [5, 6], are being advanced both theoretically and experimentally, towards the use of such non-conventional ion sources in multidisciplinary applications, including their potential use in hadrontherapy of cancer $[7,8]$. While progress towards the stringent requirements of such applications (higher energies, mono-chromaticity, controllable flux, stability and repeatability) will be facilitated by the ongoing development of next generation laser drivers and innovative target technology [9-11], present limitations in the ability to fully characterize the beams on a shot-by-shot, real time basis also need to be overcome in order to provide optimized and controllable beams for applications. An ideal diagnostic arrangement should be able to provide real-time information on basic ion beam parameters such as energy, flux, energy spread as well as on the dose delivered to an irradiated sample. Currently, standard detection systems used for characterizing such proton/ion beams can be divided into two main categories: on-line diagnostics (Thomson Parabola spectrometer, e.g. employing MCP detectors, Faraday cups (FC) and a number of other detectors working in TOF mode) and single shot passive diagnostics 45 (CR39, Radio-Chromic Films, and other types of dosimeters) [12]. The TOF technique is arguably the most established amongst the real-time diagnostics methods [13, 14, 16-19], [16, 20], and allows measuring laser-driven ion beam parameters such as ion kinetic energy, current, total charge as well as to test shot-to-shot reproducibility. Different detectors have been used so far for TOF measurements including FCs, ion collectors (IC) as well as semiconductor-like 
detectors, such as silicon carbide ( $\mathrm{SiC})$ and diamond [13, 14, 16-19]. The temporal response of FCs and ICs limits the time separation of the different forms of radiation emitted, which need to be discriminated for energy reconstruction, particularly for high-energy ( $>10 \mathrm{MeV}$ ) proton beams. SiC or diamond detectors show several advantages for TOF detection of high-energy laser-driven proton spectra. In particular, the current response of these detectors is proportional to the energy released by the incident radiation inside the detector's active layer. Consequently, one of the advantages of semiconductor-like detectors compared to other devices is their low sensitivity to plasma-emitted visible and soft-UV radiation, which damps the photo peak signal $[13,19,21]$. Thanks to this feature, coupled with the fast response typical of diamond detectors (DDs) [22] (about $400 \mathrm{ps}$ in terms of rise time for the detector used in the experiment), the fast proton signal can be easily discriminated from the photo peak allowing to observe the energy cut-off at very short TOF compared to other detectors, as reported in $[13,21,23]$. Moreover, compared to other semiconductor devices, diamond and $\mathrm{SiC}$ detectors are also more resistant to the extreme fluxes of ionizing radiation typically present in the harsh conditions of a laser-plasma environment.

The total TOF signal is generally originated from the different ion species accel70 erated from the target and reaching the detector at a specific TOF and kinetic energy. For such reason, the TOF technique is particular suitable when protons are accelerated at significantly higher energy than heavier ions (TNSA regime) and hit the detector at lower TOF than the slower ions allowing an easy discrimination of the signal uniquely originated from protons. On the other hand, for advanced acceleration regimes such as RPA from ultrathin foils, heavier ions such as Carbon can be accelerated up to similar energies per nucleon as protons and reach the detector at the same TOF, generating a unique overlapped TOF signal. Under these conditions, the proton contribution cannot be identified and disentangled from the TOF signal alone. This represents at present a limita-

so tion on the broad applicability and usage of the TOF technique as discussed in this paper. Nevertheless, innovative solutions are currently under study which 
may make TOF techniques applicable also under these advanced conditions. On

the other hand, amongst advanced acceleration settings, the TOF technique is particularly efficient when a single ion species, i.e. protons, is accelerated and detected, as in the case of cryogenic hydrogen targets.

This paper reports, for the first time, TOF measurements of laser-accelerated proton energies up to $30 \mathrm{MeV}$, performed using diamond detectors at the VULCANPW laser facility at the Rutherford Appleton Laboratory (RAL). The main goal of the measurements was to extend the range of operation of TOF-based diagnosis to tens of $\mathrm{MeV}$ and demonstrate that this approach can in principle be extended to detect proton energies up to $100 \mathrm{MeV}$. A new analysis procedure to extract energy cut-off and distributions for proton energies up to the 100 $\mathrm{MeV}$ level has been also developed as reported in [15]. The developed processing method has been also validated by comparing the energy spectra and fluence extracted from the TOF signals with the results obtained with established diagnostics tools, namely a Thomson Parabola spectrometer (TPS) and Radiochromic films (RCF).

\section{Experimental Setup}

In the experiment the VULCAN laser, of wavelength $1.054 \mu \mathrm{m}$ and a pulse tors: TPS, stacks of RCFs and a diamond detector (DD).

The TPS [25], consisting of a $1 \mathrm{~T}$ dipole magnet, electric field plates and a $400 \mu \mathrm{m}$ pinhole faced the front side of the target at a pinhole-target distance of $125 \mathrm{~cm}$ and at an angle of about $10^{\circ}$ from the target normal. An image plate duration of about 700 fs FWHM, delivered up to $400 \mathrm{~J}$ of energy on target in a single shot. The laser pulse was focused onto a $25 \mu \mathrm{m}$-thick Aluminium foil target at an incidence angle of $20^{\circ}$ with respect to the target normal, by using a f/3 off-axis parabolic mirror. The laser peak intensity was of the order of $10^{21}$ $\mathrm{W} / \mathrm{cm}^{2}$. A schematic illustration of the experimental setup is shown in Fig. 1.

The ion energy distribution was diagnosed by three different types of detec-

(IP) wrapped with a $5 \mathrm{um} \mathrm{Al}$ foil, was used as detector of the particle traces 


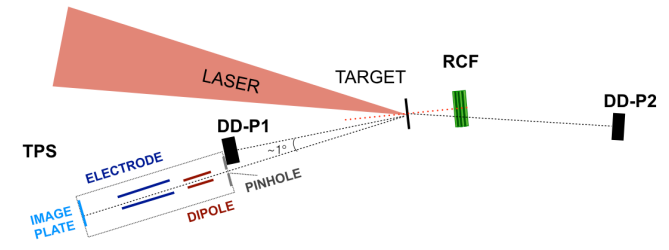

Figure 1: Experimental setup: the laser incidence angle is $20^{\circ}$, the TPS detection angle is about $10^{\circ}$ and the DD detection angle is about $10^{\circ}$ (DD has been placed alongside the TPS pinhole in the target front side direction for a first set of shots; the same DD was then moved to the target rear side in a second set of measurements)

deflected by the TPS. RCF stacks used for resolving simultaneously the spatial and energy distribution of the laser-driven proton beam, were placed at the rear side of the target at a distance of $8 \mathrm{~cm}$ along the target normal.

A polycrystal Chemical Vapour Deposition DD, $100 \mu \mathrm{m}$ thick, was used in the present experiment for the TOF diagnosis. The DD was placed at a distance of $122 \mathrm{~cm}$ (P1) from the target front-side alongside the TPS pinhole at about $10^{\circ}\left(\sim 1^{\circ}\right.$ difference can be accounted for the two diagnostics). This allowed for a direct comparison of the signals of the two diagnostics. The TPS and the DD ran simultaneously on several laser shots. In a few shots the DD was also moved to the target rear-side at a distance of $256 \mathrm{~cm}(\mathrm{P} 2)$ from the target and about $10^{\circ}$ from the target normal for a direct comparison with RCF stack measurements.

\section{Methods}

TOF technique with diamond detectors

$125 \quad$ A polycrystal $100 \mu \mathrm{m}$ thick and $2 \mathrm{~mm}$ aperture diamond detector supplied by the CIVIDEC was used during the experiment in a TOF configuration. A picture of the detector employed during the experiment (the inner part without housing) is shown in figure $2 \mathrm{a}$.

The detector housing was made of epoxy loaded FR4, gold metalized and 

size $55 \mathrm{~mm} \times 55 \mathrm{~mm} \times 15 \mathrm{~mm}$ with extra RF shielding, useful for Electromagnetic Pulse (EMP) attenuation. This solution was used as a means to optimize shielding of the electronics from the strong EMP present in high-power laser experiments. Moreover, a low-pass RC filter is integrated in the PCB board of the detectors to filter out the high frequencies (>900 MHz) typically present in the EMP spectrum, which could affect the signal acquisition.
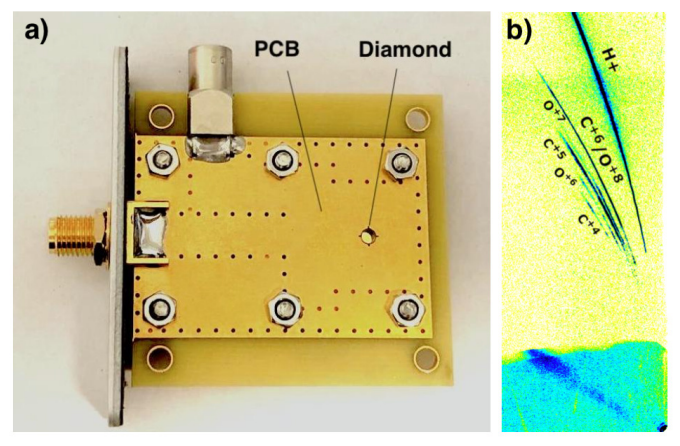

Figure 2: a) Picture of the inner structure of the detector showing the diamond and the PCB. b) Ion traces detected with the IP.

Ion current signals were stored by a $2.5 \mathrm{GHz} 40 \mathrm{Gs} / \mathrm{s}$ Tektronik digital oscilloscope with a $50 \Omega$ load impedance. Due to the very high event rate, typical of laser driven ion pulses (with peak current of the order of $10^{10}-10^{12} \mathrm{p} / \mathrm{pulse}$ ), no signal amplification is needed and the DD can be operated in current mode, i.e. the average current recorded depends on the product of the event rate and the average charge produced per event.

The start of the acquisition in the oscilloscope is typically triggered by the rise of the photo-peak signal $(\mathrm{t}=0)$. This is due to X-ray /gamma photons which are emitted from the target during the ultrashort laser -target interaction, simultaneously with the emission of the ions. However, photons travel from the target to the detector (separated by a distance L) with a finite velocity c, employing a time $\mathrm{t}_{p h}=\mathrm{L} / \mathrm{c}$ to reach the detector after they have been emitted. The time $\mathrm{t}=0$ in the oscilloscope trace corresponds to the time at which such 


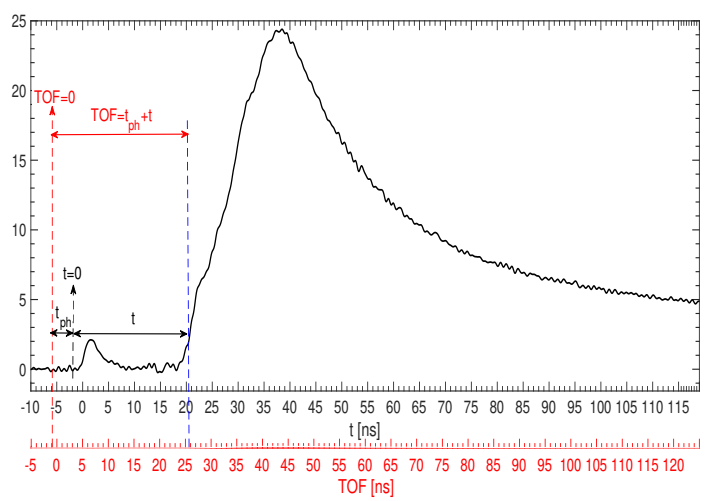

Figure 3: Time signal acquired with a fast oscilloscope triggered by the rise of the photopeak at $\mathrm{t}=0$ (black scale) and TOF scale obtained shifting the signal of $\mathrm{t}_{p h}=5.2 \mathrm{~ns}$ (red scale).

prompt $\mathrm{x} /$ gamma rays effectively reach the detector. The particle emission time, which acts as "start" for the TOF signal $(\mathrm{TOF}=0)$, therefore corresponds to the time $\mathrm{t}=-t_{p h}$ on the oscilloscope trace. In order to obtain a TOF signal from the oscilloscope trace, the time axis has to be shifted by the amount $t_{p h}$, so that the zero of the TOF temporal scale corresponds to the time at which ${ }_{55}$ the particles have been emitted from the target [21]. Figure 3 shows a typical signal as acquired from a diamond detector $(\mathrm{L}=1.5 \mathrm{~m})$ in the oscilloscope with the rise of the photopeak acting as trigger at $t=0$ (black scale) and the shifted TOF scale obtained accounting for $\mathrm{t}_{p h}$ (red scale).

$T P S-I P$

The Thomson Parabola Spectrometer used in the experiment was composed of a $5 \mathrm{~cm}$ thick $1 \mathrm{~T}$ dipole and a pair of electric plates charged at $20 \mathrm{KV}$. BAS-TR image plates (IP) [27] were used as detector for the particle traces and placed at about $50 \mathrm{~cm}$ from the magnet entrance. The IPs were wrapped with $5 \mu \mathrm{m}$ thick $\mathrm{Al}$ foils to avoid their exposure to ambient light after irradiation. They were scanned after few hours from irradiation with a commercial IP scanner (Fujifilm FLA-500025), with a 16-bit dynamic range and a $25 \mu \mathrm{m} \times 25 \mu \mathrm{m}$ pixel size. The measured signal was converted from the pixel value measured with the 
scanner to PSL (photo-stimulated luminescence) using the formula given by the manufacturer Fujifilm IP . PSL is then converted into fluence for energy unit and for a specific ion species, through a calibration providing the response of the IP to a specific type of radiation with known intensity and energy [28-30]. Knowing the specific geometric configuration, as well as the strength of the magnetic and the electric fields of the TPS, it is then possible to identify the ion species corresponding to a given trace and reconstruct the energy spectrum by converting the measured PSL into particle number via the calibration. A raw image of the IP acquired during one shot is shown in figure $2 \mathrm{~b}$. In this case carbon and oxygen ions are identified with the following charge states: $\mathrm{C}^{6+}$, $\mathrm{C}^{5+}, \mathrm{C}^{4+}, \mathrm{O}^{8+} . \mathrm{O}^{7+}$. Using the IP calibration in [30], the energy spectrum for carbon ions can be reconstructed revealing a maximum energy of $\sim 4 \mathrm{MeV} / \mathrm{u}$ for $\mathrm{C}^{6+}$. Such information was used for the TOF analysis.

$R C F s$

Two different types of RCF were used for the RCF stacks: the less sensitive HD-V2 type for low energy proton and the more sensitive EBT-3, used to detect the high-energy cut-off of the spectrum. The RCF layers were also alternate with Mylar and copper foils hundreds $\mu \mathrm{m}$ thick to increase the signal on the RCFs maintaining an high sensitivity at high energy. The $5 \times 5 \mathrm{~cm}^{2}$ RCF stacks were placed along the target normal direction on the rear side at about $8 \mathrm{~cm}$ from the target. A $12 \mu \mathrm{m} \mathrm{Al}$ foil was used to wrap the stack in order to shield the RCF from x-rays. The RCF images were acquired using a commercial V750 EPSON scanner, and a dose calibration performed with conventionally accelerated protons with an energy comparable with the energy achieved in the present experiment, was used to convert the pixel value in absolute dose, as needed for the absolute fluence calculation.

The proton energy spectrum is then obtained recording the dose deposited in each RCF layer and using an iterative routine taking into account of the proton depth-dose profile along the RCF stack as it is explained in [31]. As an example, figure 4 shows the images of the first 4 layers (HD-V2 type) of a 


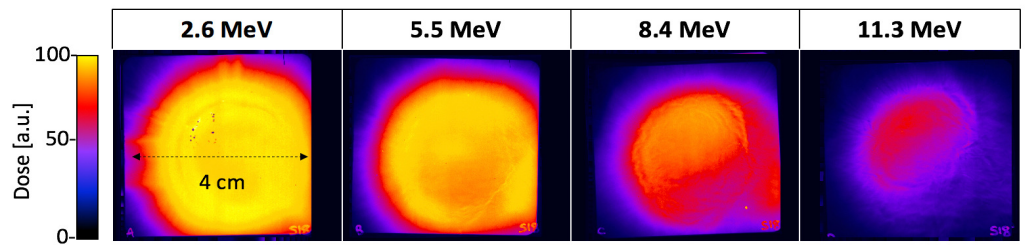

Figure 4: First 4 layers of the RCF stack irradiated during one shot. The proton energies stopped in each layer are also indicated.

RCF stack irradiated during a single shot. A $4 \mathrm{~cm}$ beam full diameter can be measured in the first layer of the stack, corresponding to a maximum divergence for 2.6 MeV protons of $\pm 15^{\circ}$. A smaller region of interest (ROI) with a 1-1.5 $\mathrm{mm}$ radius has been then chosen for the dose analysis of each RCF stack layer, corresponding to a solid angle of about $10^{-4}$ sr. In order to obtain the proton energy spectrum along the same direction of the DD for comparison, i.e. $10^{\circ}$ with respect to the target normal, such ROI was chosen over the RCF region corresponding to the target-DD direction, i.e. about $1.4 \mathrm{~cm}$ off axis.

\section{Results and discussion}

Fig 5a shows a typical TOF signal acquired by the DD placed in the target front side direction at a distance of $122 \mathrm{~cm}(\mathrm{P} 1)$. A $50 \mu \mathrm{m} \mathrm{Al}$ foil was used in front of the DD aperture in order to stop low energy protons and filter out the heavier ions present in the beam. The small peak, observed at about 5 ns, corresponds to the so-called photo-peak, generated by the hard X-rays flash component followed by hot plasma electrons. A narrow peak, rising sharply at about $20 \mathrm{~ns}$ and corresponding to the highest energy protons can be clearly discriminated from the photo peak. Based on the TPS-IP analysis (see above), carbon and oxygen ions with maximum energies of about $4 \mathrm{MeV} / \mathrm{u}$ were accelerated together with protons. In agreement with simulations carried out using the LISE $^{++}$software $\mathrm{C}$ and $\mathrm{O}$ ions with energies up to about $4 \mathrm{MeV} / \mathrm{u}$ are stopped inside the $50 \mu \mathrm{m} \mathrm{Al}$ absorber and do not contribute to the TOF signal observed. 
Protons with energies lower than about $2 \mathrm{MeV}$, corresponding to TOF values
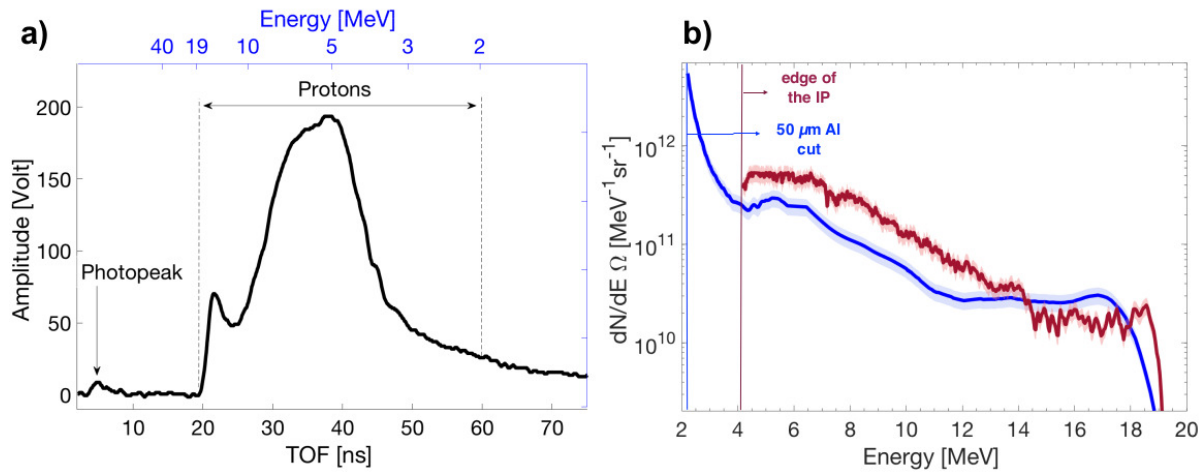

Figure 5: a) Typical TOF signal acquired by the DD, in backward direction, at a distance from the target of $122 \mathrm{~cm}$. b) Proton energy spectra reconstructed from the DD TOF signal in the interval $20 \mathrm{~ns} \leq \mathrm{TOF} \leq 60 \mathrm{~ns}$ (blue line) and using the TPS-IP (red line) for the same shot.

Looking at the TOF signal in figure 5, two peaks at TOF around $25 \mathrm{~ns}$ and $35 \mathrm{~ns}$ are well visible, both attributed to the protons accelerated from the target. It is worth to highlight that the temporal structures in the TOF signals, resulting as local peaks at certain TOF values, do not necessarily correspond to peaks in particle flux and intensity. As it will be explained in details in the next paragraphs, the signal generation in semiconductor-like detectors used in TOF configuration depends both on the intrinsic temporal response of the detector and on its response to particles, depending in turn on the particle flux at a specific TOF and on the corresponding energy loss in the detector's active layer $[19,23]$. Figure 5 also shows a tail in the TOF signal for TOF $>60 \mathrm{~ns}$, which, due to the filtering, cannot be due to direct particle detection. We believe that this is due to the detector discharging after being irradiated with a large particle flux as well as to saturation effects in the charge collection. For this reason, $\mathrm{TOF}=60 \mathrm{~ns}$ was the maximum $\mathrm{TOF}$ we considered in the procedure to convert the TOF signal in proton energy spectrum.

The incident ion energy can be calculated from the TOF values by taking into 
account the distance travelled from the target to the detector by the X-ray flash as well as the flight path and mass of the ion of interest. The short duration ( $\sim 5 \mathrm{~ns}$ ) and low amplitude of the photopeak are crucial features enabling the detection of the minimum TOF (maximum energy) of protons with energies up to several tens of $\mathrm{MeV}$. For these energies the time interval between the photo peak and the fast ion signal is minimized and the latter could in principle overlap with a longer photo-peak using different detectors (typical photopeak duration measured by FCs and ICs ranges between $50 \mathrm{~ns}$ and $100 \mathrm{~ns}$ at $1.5 \mathrm{~m}$ from the target). In Fig. 5a the sharp peak, attributed to fast $\mathrm{H}^{+}$signal, is however well separated from the photo peak and a proton energy cut-off of $19 \mathrm{MeV}$, corresponding to a TOF value of $20 \mathrm{~ns}$, can be easily measured even at the relatively short distance of $1 \mathrm{~m}$ from the target. The proton energy distribution was reconstructed from the TOF amplitude taking into account the DD response and using the approach described in [14]. The DD current response depends on the energy deposited in the detector thickness, and thus, for ion energies up to few $\mathrm{MeV}$, the ion range is comparable with the active thickness, ions are stopped inside the active layer and the energy deposited inside the detector corresponds to the incident kinetic energy. On the other hand, ions with energies higher than about $5 \mathrm{MeV}$ have sufficient kinetic energy to traverse the detector thickness and therefore, in order to reconstruct the ion energy distribution from the TOF amplitude, the variation of the energy loss inside the active layer needs to be taken into account. The new analysis procedure developed and described in details in [15] allows to extract the proton energy distribution from TOF signals under these conditions. Once the incident energy is calculated from the TOF data, a Monte Carlo (MC) simulation is used to estimate the energy deposited inside the detector thickness for a given incident energy. A proper estimation of the energy loss inside the detector active thickness as a function of the incident energy allows attributing the correct proton yield to the TOF amplitude and, as a consequence, the energy spectrum can be extracted.

Fig 5b shows the proton energy distribution extracted applying this data processing method on the TOF signal, shown in Fig. 5a, in the interval 20 ns-60 
ns. Fig 5b also shows a comparison between the proton energy distribution extracted from the DD1 and the one obtained from the TPS for the same shot. A good quantitative agreement can be observed. The cut off energies measured, respectively, from the TOF and the TP energy distributions are 19.4 $\pm 0.2 \mathrm{MeV}$ and 19.2 $\pm 0.2 \mathrm{MeV}$. Furthermore, a comparison between the number of protons per solid angle obtained integrating the TOF and the TP energy spectra between 4 and $19 \mathrm{MeV}$, results respectively in $1.3 \pm 0.3 \times 10^{12} \mathrm{p} / \mathrm{sr}$ and $2.4 \pm 0.5 \times 10^{12} \mathrm{p} / \mathrm{sr}$, which is still in reasonable agreement considering the slightly different angular position of the TOF detector and the TPS pinhole. Such technique was used in several shots throughout the experiment to recover the maximum proton energies and the absolute number of protons in a given energy interval allowing to monitor the shot-to-shot reproducibility. As an example, figure 6 and figure 7 show respectively the proton energy cut-offs along with the corresponding number of protons per solid angle $(\mathrm{Np} / \mathrm{sr})$ in the energy interval between 10 and 18 $\mathrm{MeV}$, for 7 shots performed under the same nominal conditions (DD placed in $\mathrm{P} 1)$.

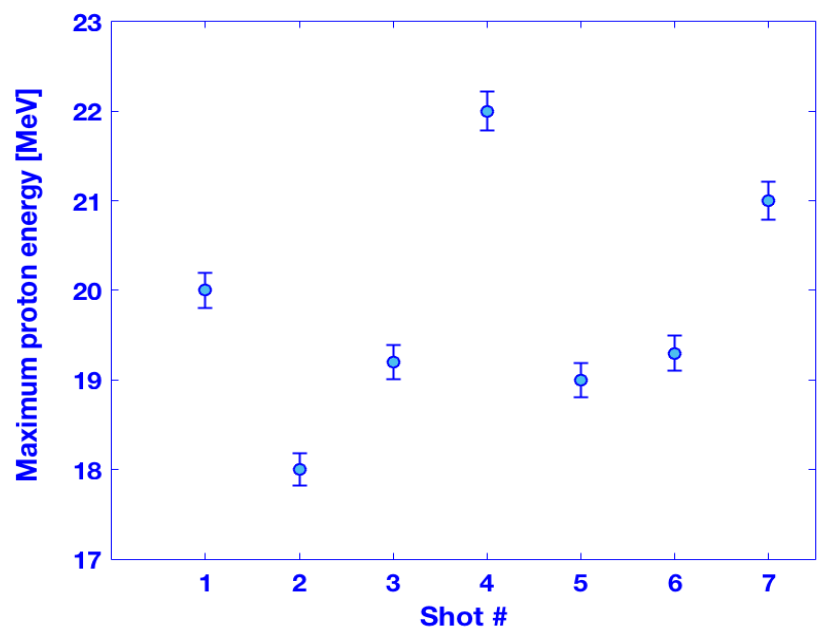

Figure 6: Maximum proton energies measured with the DD detector placed in P1 for 7 shots.

TOF technique proved to be sensitive to small fluctuations in energy and 


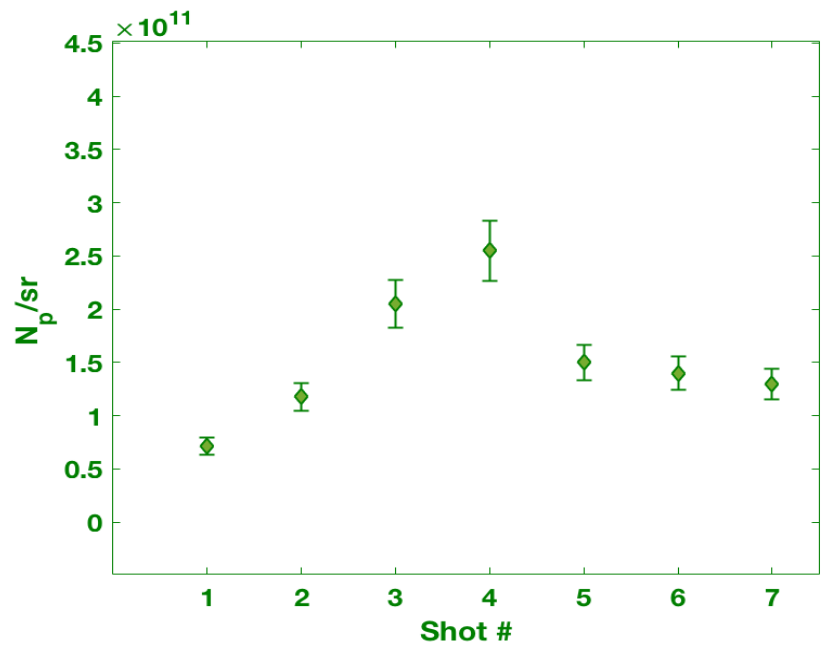

Figure 7: Number of protons per solid angle within the energy interval [10-18] MeV measured with the DD detector placed in P1 for 7 shots. 
to the target-DD direction. In this configuration, an average maximum proton energy over several shots, of $31.8 \pm 0.6 \mathrm{MeV}$ with a flux at the cut-off of $5 \cdot 10^{9}$ $\mathrm{MeV}^{-1} \mathrm{Sr}^{-1}$ was obtained. As an example, the proton energy spectrum measured with a RCF stack (higher energy) during one single shot is shown in the inset of figure $8 \mathrm{~b}$ in comparison with the one reconstructed from the TOF signal.

Fig. 8 shows the TOF spectrum measured by the DD placed in the laser forward direction at a distance of $256 \mathrm{~cm}$ from the target (P2) and about $10^{\circ}$ from the target normal. The TOF spectrum shows two main ion groups in addition to the short photo-peak lasting about 5 ns. The fast ion peak (34 ns-55 ns) can be mainly attributed to $\mathrm{H}^{+}$and the broad structure (55 ns-130 ns) arises from the overlap between the signals generated by the higher- $\mathrm{Z}$ target contaminants (mainly $\mathrm{C}$ and $\mathrm{O}$ ions) and the signal due to the slow $\mathrm{H}^{+}$group.
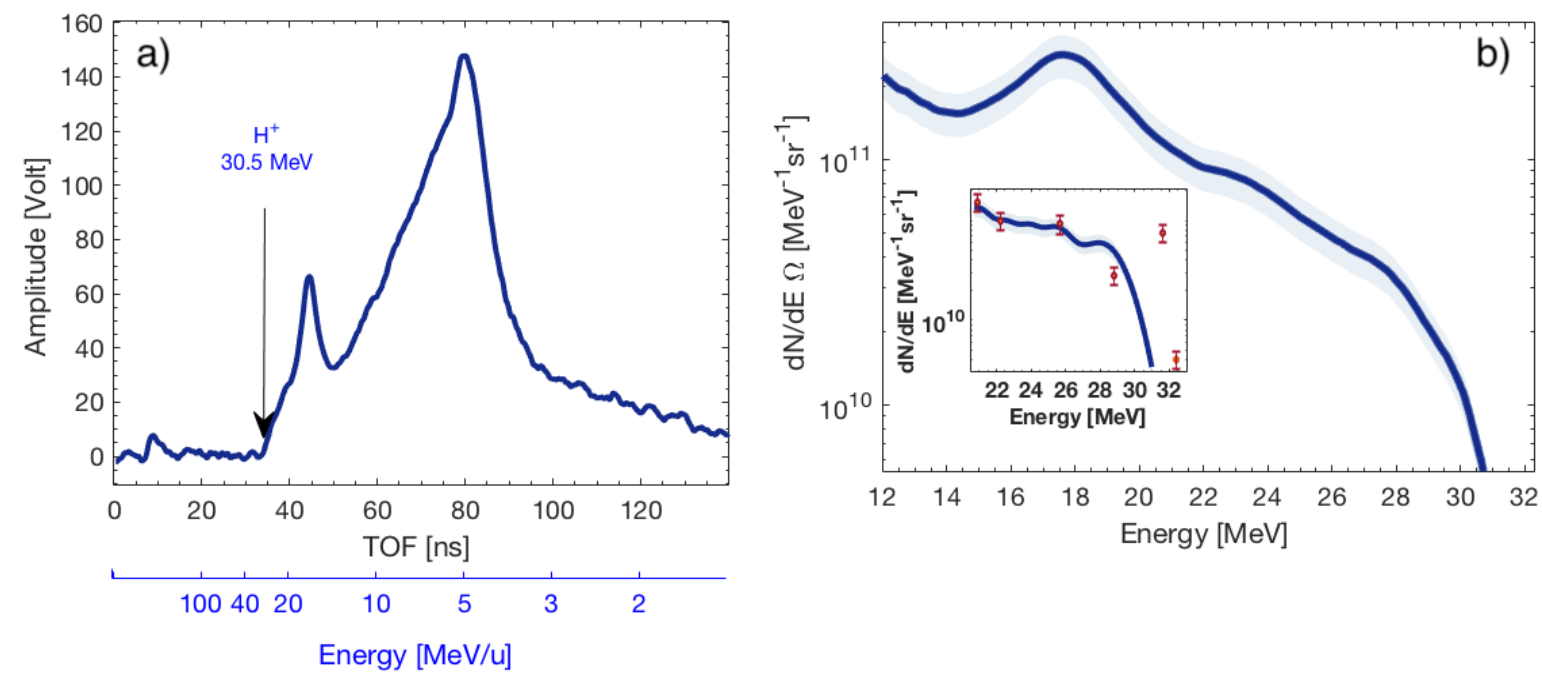

Figure 8: a)Typical TOF signal acquired by DD in forward direction at $256 \mathrm{~cm}$ from the target and b) corresponding proton energy spectrum. The inset shows the proton energy distribution extracted from the TOF data between 34 ns and 55 ns (blue line) compared with the one measured with RCF stack (red diamond).

In this experiment, the ion beam emitted in the laser forward direction was not diagnosed using a TPS, thus speculations about the species, charge states 
and maximum energies of heavier ions accelerated from the target rear-side cannot be confirmed by TPS ion spectra. Nevertheless, on the basis of Thomson spectra obtained in other experimental run on the VULCAN PW system with similar targets and laser conditions, we can assume that the maximum energy per nucleon of Carbon/Oxygen ions accelerated in the forward direction will be below $10 \mathrm{MeV} / \mathrm{u}$ [25]. On this basis, with reasonable confidence, the TOF signal acquired for proton energies higher than about $10 \mathrm{MeV}$, which corresponds to TOF shorter than about $60 \mathrm{~ns}$, can be uniquely attributed to $\mathrm{H}^{+}$. As one can see in Fig.8, the fast proton signal is strongly emphasized, as it is expected considering the dependence of the DD response from the energy deposited inside the active thickness. This feature coupled with the fast time response and high signal-to-noise ratio characteristics of these detectors allows determining the energy of the ions with a very high resolution. In particular, it allows to discriminate the fastest proton component of the beam from the photo-peak as well as from the slower ion contribution, even at a relatively short distance from the source. In Fig 8 a minimum TOF value of 34 ns can be clearly identified allowing the measurement of a maximum proton energy of $31 \mathrm{MeV}$. To our knowledge the proton energy cut-off measured in the present experiment is the highest proton energy measured using the TOF technique to date. The highenergy proton spectrum extracted from TOF data between 34 ns and 55 ns is shown in the inset in fig. 8. The proton flux measured at the cut-off energy $(30.5 \pm 0.5 \mathrm{MeV})$ is $\sim 5 \cdot 10^{9} \mathrm{MeV}^{-1} \mathrm{sr}^{-1}$, in very good agreement with the RCF data. These results confirm that proton energies of several tens of $\mathrm{MeV}$ can be easily measured in real time using the TOF technique coupled with diamond detectors. The result obtained also indicates that this diagnostics could be successfully used to measure proton energy cut-offs of the order of $100 \mathrm{MeV}$ (as recently reported in experiments on the same facility [26]) at relatively short detection distances. The TOF spectrum in Fig 8 clearly shows that the TOF signal of $100 \mathrm{MeV}$ protons, which corresponds to $19.5 \mathrm{~ns}$ assuming a flight path of $2.5 \mathrm{~m}$, could still be well separated in time from the photopeak. Moreover, the TOF technique could be used for the diagnosis of much higher energy protons, 
e.g. the $250 \mathrm{MeV}$ typically required for cancer therapy, by suitably increasing the flight path. A slightly increased flight path of $3 \mathrm{~m}$ would allow detecting $250 \mathrm{MeV}$ protons at a TOF of about $16 \mathrm{~ns}$, still distinguable from a typical photopeak acquired with such type of detector as shown in Fig 8.

\section{Conclusion}

The use of real time diagnosis based on the TOF technique using diamond detectors for full characterization of high-energy protons has been reported for the first time for ions accelerated by a kJ-class, PW laser system. Proton cut-off energies, exceeding $30 \mathrm{MeV}$, have been measured with this diagnostics technique at a detection distance of about $2.5 \mathrm{~m}$; such results point out that proton cutoff energies up to $100 \mathrm{MeV}$ can be detected using TOF diamond detectors even at short distances from the target. In addition, proton energy distributions have been also extracted from the TOF signal and the total proton number per steradians has been estimated in a broad energy range (from few $\mathrm{MeV}$ up to about $30 \mathrm{MeV}$ ) thanks to a new analysis method developed for data processing of high-energy ion TOF signal.

The TOF detection system, presented here, can be used to monitor on a shot-toshot basis the main ion beam features and is capable of operating at a repetition rate of $10 \mathrm{~Hz}$. It will be used to optimize the ion beam transport and selection and tune in real-time the particle beam transport optics along the user beam line ELIMAIA (ELI Multidisciplinary Applications of laser-Ion Acceleration), which has been installed at the ELI-Beamlines facility in Prague (Czech Republic) in 2018 [24]. This diagnostic approach is particularly attractive for the characterization of sources of single species ion beams, e.g. as emerging from cryogenic hydrogen target experiments $[10,32,33]$.

\section{Acknowledgments}

This work has been supported by the ELIMED activities funded by the $\mathrm{V}$ committee of INFN (Italian Institute for Nuclear Physics), by the MIUR (Ital- 
funded by the Ministry of Education, Youth and Sports as part of targeted support from the National Programme of Sustainability II, by the project Advanced research using high intensity laser produced photons and particles (CZ.02.1.01/0.0/0.0/16_019/0000789) from European Regional Development Fund

ian Ministry of Education, Research and University), by the Project LQ1606 (ADONIS) and by EPSRC (grants n. EP/K022415/1 and and EP/P010059/1). The work has also received funding from the EMPIR programme co-financed by the Participating States and from the European Union's Horizon 2020 research and innovation programme. We also ackowledge support from the staff of the Central Laser Facility at the Rutherford Appleton Laboratory.

\section{References}

[1] Danson, C., et al., Petawatt and exawatt class lasers worldwide, High Power Laser Science and Engineering, vol. 7, p. e54, 2019

[2] Wilks, S. C. , Langdon, A. B., Cowan, T. E., Roth,M. , Singh,M. , Hatchett,S., Key,M.H., Pennington, D., MacKinnon, A., \& Snavely,R. A., Energetic proton generation in ultra-intense laser-solid interactions. Phys Plasmas 8, 542-9 (2001).

[3] Passoni, M., Bertagna, L., Zani, A., Target normal sheath acceleration: Theory, comparison with experiments and future perspectives. New J Phys

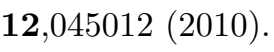

[4] Macchi, A., Borghesi, M., Passoni, M., Ion acceleration by superintense laser-plasma interaction. Rev Mod Phys 85, 751-93 (2013).

[5] Macchi, A., Veghini, S., Liseykina, T. V., Pegoraro, F., Radiation pressure acceleration of ultrathin foils.New J Phys 12, 045013 (2010).

[6] Kar, S. et al., Ion acceleration in multispecies targets driven by intense laser radiation pressure. Phys Rev Lett 109, 185006 (2012). 
[7] Bulanov, S.V., Khoroshkov, V.S., Feasibility of using laser ion accelerators in proton therapy. Plasma Phys Reports 28, 453-6 (2002).

[8] Bulanov, S V., Laser ion acceleration for hadron therapy.Proc - 2014 Int Conf Laser Opt LO 2014 57, 1149-79 (2014).

[9] Margarone, D. , Cirrone, G.A.P., Cuttone, G. , Korn, G., Preface: 2nd ELIMED workshop and panel. AIP Conf. Proc. 546 (2013).

[10] Margarone, D. , et al., Proton acceleration driven by a nanosecond laser from a cryogenic thin solid-hydrogen ribbon. Phys Rev X 6, 041030 (2016).

[11] Kar, S., et al., Guided post-acceleration of laser-driven ions by a miniature modular structure. Nat Commun 7, 10792 (2016).

[12] Bolton, P.R., et al., Instrumentation for diagnostics and control of laseraccelerated proton (ion) beams. Phys Medica 30, 255-70 (2014).

[13] Margarone, D., et al., Diamond detectors for characterization of lasergenerated plasma. Radiat Eff Defects Solids 163, 463-70 (2008).

${ }_{415}^{4}$ [14] Margarone, D., et al., Full characterization of laser-accelerated ion beams using Faraday cup, silicon carbide, and single-crystal diamond detectors. $J$ Appl Phys 109, 103302 (2011).

[15] Milluzzo, G., et al., A new energy spectrum reconstruction method for Time-Of-Flight diagnostics of high-energy laser-driven protons, Rev. Sci. Instrum 90, 083303 (2019).

[16] Prokůpek, J., et al., Experimental test of TOF diagnostics for PW class lasers.SPIE Proceedings $\mathbf{8 7 7 9}$ (2013).

[17] Marinelli, M., et al., Analysis of laser-generated plasma ionizing radiation by synthetic single crystal diamond detectors. Appl Surf Sci 272, 104-8 (2013). 
[18] Bertuccio, G., Puglisi, D. ,Torrisi, L., Lanzieri, C. , Silicon carbide detector for laser-generated plasma radiation. Appl Surf Sci 272, 128-31 (2013).

[19] Milluzzo, G., et al., TOF technique for laser-driven proton beam diagnostics for the ELIMED beamline. J Instrum 12, C03044 (2017).

[20] Picciotto, A., et al., Microfabrication of Silicon Hydrogenated Thin Targets for Multi-MeV Laser-Driven Proton Acceleration. Appl Phys Express 4, 126401 (2011).

[21] Scuderi, V., et al., Time of Flight based diagnostics for high energy laser driven ion beams. J Instrum 12, C03086 (2017).

[22] Randazzo, N.,et al., Comparative timing performances of S-CVD diamond detectors with different particle beams and readout electronics. Nuclear Science Symposium and Medical Imaging Conference (NSS/MIC) IEEE 10.1109/NSSMIC.2012.6551450 (2012).

[23] Milluzzo, G., et al., Laser-accelerated ion beam diagnostics with TOF detectors for the ELIMED beam line. J Instrum 12, C02025 (2017).

[24] Scuderi, V., et al., Diagnostics and Dosimetry Solutions for Multidisciplinary Applications at the ELIMAIA Beamline. Appl Sci 8, 1415 (2018).

[25] Alejo, A.,et al., High resolution Thomson Parabola Spectrometer for full spectral capture of multi-species ion beams. Rev Sci Instrum 87, 083304 (2016).

[26] Higginson, A., et al., Near-100 $\mathrm{MeV}$ protons via a laser-driven transparency-enhanced hybrid acceleration scheme. Nat Commun 9, 724 (2018).

[27] Bonnet, T.,et al., Response functions of Fuji imaging plates to monoenergetic protons in the energy range $0.6-3.2 \mathrm{MeV}$, Rev. Sci. Instrum. 84, 013508 (2013). 
[28] Mancic, A. ,et al., Absolute calibration of photostimulable image plate detectors used as $(0.5-20 \mathrm{MeV})(0.5-20 \mathrm{MeV})$ high-energy proton detectors, Review of Scientific Instruments 79, 073301 (2008);

[29] Rabhi, N., et al., Calibration of imaging plate detectors to mono-energetic protons in the range 1-200 MeV, Review of Scientific Instruments 88, 113301 (2017);

[30] Doria, D., et al., Calibration of BAS-TR image plate response to high energy (3-300 MeV) carbon ions, Review of Scientific Instruments 86, 123302 (2015).

[31] Kirby, D.,et al., Radiochromic film spectroscopy of laser-accelerated proton beams using the FLUKA code and dosimetry traceable to primary standards. Laser and Particle Beams 29(2), 231-239.

[32] Polz, J., Robinson, A.P.L., Kalinin, A. et al., Efficient Laser-Driven Proton Acceleration from a Cryogenic Solid Hydrogen Target. Sci Rep 9, 16534 (2019).

[33] Obst, L., Göde, S., Rehwald, M. et al., Efficient laser-driven proton acceleration from cylindrical and planar cryogenic hydrogen jets. Sci Rep 7, 10248 (2017). 